\title{
On the orbital magnetic moment and anisotropy energy of the ordered $\mathrm{Fe}_{0.5} \mathrm{Pd}_{0.5}$ alloy
}

\author{
G.Y. Guo ${ }^{\mathrm{a}, \mathrm{b}, *}$, Y.L. Wang ${ }^{\mathrm{b}}$, C.T. Chen ${ }^{\mathrm{b}}$ \\ ${ }^{a}$ Department of Physics, National Taiwan University, Taipei 106, Taiwan \\ ${ }^{\mathrm{b}}$ Synchrotron Radiation Research Center, Hsinchu 300, Taiwan
}

\begin{abstract}
We haved calculated the magnetic moments and magnetic anisotropy energy (MAE) of the ordered $\mathrm{Fe}_{0.5} \mathrm{Pd}_{0.5}$ alloy by using relativistic linear muffin-tin orbital and full-potential linear augmented plane wave methods and also including the orbital polarization correction (OPC) and the generalized gradiant approximation. The OPC substantially increases the $\mathrm{Fe}$ orbital moments in the alloy, thereby reducing the discrepancy between the measured and theoretical orbital moments. For all the lattice parameters considered, the [0 01 1] direction is found to be the easy axis, thus showing no spin-reorientation transition as suggested recently. The calculated MAE agrees with experiments. (C) 2002 Elsevier Science B.V. All rights reserved.
\end{abstract}

Keywords: Magnetic moments; Magnetocrystalline anisotropy

The $\mathrm{Fe}_{0.5} \mathrm{Pd}_{0.5}$ alloy exhibits a phase transition around $920 \mathrm{~K}$ between a chemically disordered FCC phase and an ordered $L 1_{0}$ tetragonal structure consisting of alternating planes of $\mathrm{Fe}$ and $\mathrm{Pd}$ atoms [1]. This chemical ordering gives rise to enhanced Kerr rotations and also a strong magnetocrystalline anisotropy [2,3]. Furthermore, the $L 1_{0}$ phase thin films were recently fabricated by molecular beam epitaxy [4] with varying lattice parameters depending on the substrates and growth conditions. Clearly, these films with a strong perpendicular magnetic anisotropy (PMA) are of considerable interest for magneto-optical recording media. Therefore, $\mathrm{Fe}_{0.5} \mathrm{Pd}_{0.5}$ alloy in bulk or film has been extensively studied (e.g., Refs. [2-7]). The magnetic moments and anisotropy energy of the pseudocubic $L 1_{0}$ structure were calculated from first-principles before [5,6]. More recently, Galanakis et al. [8] calculated the magnetic properties of the ordered $\mathrm{Fe}_{0.5} \mathrm{Pd}_{0.5}$ alloy for a range of lattice parameters. Surprisingly, Galanakis et al. reported that the inclusion of the orbital-polarization correction (OPC) [9] scarcely changes the orbital

*Corresponding address: Department of Physics, National Taiwan University, Taipei 106, Taiwan. Fax: +886-223639984.

E-mail address: gyguo@phys.ntu.edu.tw (G.Y. Guo). magnetic moment of $\mathrm{Fe}$ in the $\mathrm{Fe}_{0.5} \mathrm{Pd}_{0.5}$ alloy, in contradiction to previous calculations (e.g., Refs. [5,10]). It is well known that though local-spin density approximation (LSDA) calculations describe well the spin magnetic moments in many solids, they often give too small orbital moments. A solution to this defect is to include in the Kohn-Sham equation an orbital-polarization correction (OPC) [9] of $-B L_{z}^{\sigma}$ where $B$ is the Racah parameter (for d states) and $L_{z}^{\sigma}$, the total orbital momentum for spin $\sigma$. The OPC has been found to bring the calculated orbital moments in many metallic solids in good agreement with experiments (e.g., Refs. [9,10]). Galanakis et al. also reported that the use of the generalized gradient approximation (GGA) changes the theoretical magnetocrystalline anisotropy energy (MAE) enormously. For example, the MAE calculated with GGA for the elongated face-centered tetragonal (EFCT) structure is one order of magnitude larger than that obtained with LSDA, and for the compressed facecentered tetragonal (CFCT) structure the use of GGA even gives an in-plane magnetization. As a consequence, Galanakis et al. predicted that there would be a spinreorientation transition as a function of the lattice constant $a$ and the $c / a$ ratio. This interesting magnetic transition was, however, found to be absent in their LSDA calculations. 
We have performed a careful study of the magnetic moments and MAE of the ordered $\mathrm{Fe}_{0.5} \mathrm{Pd}_{0.5}$ alloy within LSDA with and without the use of GGA as well as with and without OPC. We used both fully-relativistic linear muffin-tin orbital (LMTO) method [11] and also full-potential linear augmented plane wave (FLAPW) method [12]. The purpose of this short paper is primarily to report that the inclusion of OPC increases significantly the orbital moment of $\mathrm{Fe}$ in $\mathrm{Fe}_{0.5} \mathrm{Pd}_{0.5}$ (Table 1), in disagreement with Galanakis et al. [8] but in broad agreement with other previous calculations $[9,10]$, and that the use of GGA does not change the calculated MAE significantly (Table 2), again in disagreement with Ref. [8]. In particular, we do not find the spin reorientation reported by Galanakis et al. [8]. Finally, unlike Ref. [8], our calculated MAE with OPC for the CFCT structure is in good agreement with experiments [2,3] (Table 2).

We first performed self-consistent spin-polarized relativistic electronic structure calculations for the EFCT, FCC and CFCT structures using the LMTO method [11]. The lattice parameters used (Table 1) are the same as that for the corresponding structures in Ref. [8]. The basis functions for all the atoms used are s, $\mathrm{p}, \mathrm{d}$ and $\mathrm{f}$ MTO's. The Fe atomic sphere radius used is 2.662 a.u. for bulk BCC Fe and that of Pd is 2.948 a.u. for EFCT, 2.937 a.u. for FCC and 2.927 a.u. for CFCT. The number of $k$-points in the irreducible wedge (IW) of the Brillouin zone (BZ) is around 2000 for all three systems. In these self-consistent calculations, the magnetization is assumed to be perpendicular to the $\mathrm{Fe}(\mathrm{Pd})$ layers (i.e., along [ $\left.\left[\begin{array}{ll}0 & 1\end{array}\right]\right)$. We have considered four cases, namely, (a) LSDA, (b) LSDA plus GGA (i.e., GGA), (c) LSDA plus OPC (LSDA-OPC) and (d) GGA plus OPC (GGA-OPC). We have used the accurate local exchange- correlation potential parametrized by Voskot et al. [13] in the cases of $(\mathrm{a}, \mathrm{c})$ and the GGA generated by Perdew et al. [14] in the cases of (b,d). After the self-consistent calculations, the MAE was then calculated as the difference in the eigenvalue sum between the magnetization along [ 100$]$ and [0 011$]$. A much finer mesh over the IWBZ (2/16 of BZ) with around $13000 k$-points was used. With this choice of the number of $k$-point, the calculated MAE was found to be converged well within $5 \%$.

The calculated magnetic moments for Fe and Pd in in all three structures for the magnetization along [ $\left[\begin{array}{lll}0 & 1\end{array}\right]$ are listed in Table 1. Clearly, Table 1 shows that in all the cases, the inclusion of OPC increases the $\mathrm{Fe}$ orbital moment substantially (by $60 \sim 80 \%$ ). This significant effect of OPC on the orbital moments has been reported for other metallic systems in previous papers (e.g., $[5,10])$ using different methods. It is gratifying that for the ordered $\mathrm{Fe}_{0.5} \mathrm{Pd}_{0.5}$ alloy in the CFCT structure, the computed Fe orbital moment in the LSDA and GGA calculations (Table 1) are in good agreement with that [0.09 $\mu_{\mathrm{B}}$ (LSDA) and $0.15 \mu_{\mathrm{B}}$ (LSDA-OPC)] obtained by Daalderop et al. [5]. Thus, it is found somewhat strange that Galanakis et al. [8] reported almost zero effect of OPC on the orbital moments.

Nonetheless, the $\mathrm{Fe}$ orbital moment of $0.15 \mu_{\mathrm{B}}$ computed with OPC in the CFCT structure is still considerably smaller than that (about $0.4 \mu_{\mathrm{B}}$ ) measured by Kamp et al. [4] using X-ray magnetic circular dichroism (XMCD) technique. However, since the absolute values given by the XMCD technique depend on a number of parameters used (e.g., the number of dholes, the cut-off energy and the backgroundsubstraction) $[7,4,15]$ and since the orbital moments computed with OPC have been found to be in good agreement with

Table 1

Calculated spin $\left(m_{\mathrm{s}}\right)$ and orbital $\left(m_{\mathrm{o}}\right)$ (in brackets) magnetic moments (in units of $\left.\mu_{\mathrm{B}}\right)$ of $\mathrm{Fe}$ and $\mathrm{Pd}$ in the ordered $\mathrm{Fe}_{0.5} \mathrm{Pd}_{0.5}$ alloy in the elongated (EFCT), ideal (FCC) and compressed (CFCT) $L 1_{0}$ structures. $M_{\mathrm{t}}$ denotes the total magnetic moment per unit cell. The structural parameters $(a, c / a)$ used are also listed. Expt. denotes the measured value from Refs. [2,3]

\begin{tabular}{lllll}
\hline & Atom & EFCT & FCC & CFCT \\
\hline$a(c / a)$ & & $3.73 \AA(1.046)$ & $3.8 \AA(1.0)$ & $3.89 \AA(0.938)$ \\
Expt. & $M_{\mathrm{t}}$ & & & $3.12(3.28)$ \\
LSDA & $\mathrm{Fe}$ & $2.94(0.083)$ & $2.96(0.083)$ & $3.01(0.086)$ \\
& $\mathrm{Pd}$ & $0.27(0.023)$ & $0.30(0.023)$ & $0.32(0.024)$ \\
LSDA-OPC & $M_{\mathrm{t}}$ & 3.32 & 3.37 & 3.44 \\
& $\mathrm{Fe}$ & $2.94(0.134)$ & $2.96(0.142)$ & $3.01(0.155)$ \\
GGA & $\mathrm{Pd}$ & $0.27(0.021)$ & $3.43(0.025)$ & $0.32(0.026)$ \\
& $M_{\mathrm{t}}$ & 3.36 & $3.04(0.082)$ & 3.51 \\
GGA-OPC & $\mathrm{Fe}$ & $3.04(0.081)$ & $0.28(0.023)$ & $3.10(0.084)$ \\
& $\mathrm{Pd}$ & $0.28(0.023)$ & 3.43 & $0.30(0.025)$ \\
& $M_{\mathrm{t}}$ & 3.42 & $3.04(0.133)$ & 3.51 \\
& $\mathrm{Fe}$ & $3.04(0.133)$ & $0.28(0.026)$ & $3.10(0.146)$ \\
& $\mathrm{Pd}$ & $0.28(0.026)$ & 3.48 & $0.30(0.027)$ \\
\hline
\end{tabular}


experiments for many bulk systems $[5,15,10]$, the large discrepancy between the present OPC results and the XMCD measurements [4] might partly be attributed to the uncertainties associated with the application of the XMCD technique to this particular system. Note that the $\mathrm{Fe}$ spin magnetic moment $\left(2.04 \mu_{\mathrm{B}}\right)$ reported by Kamp et al. [4] using the same technique is also considerably smaller than the present and previous theoretical results (see Table 1 and also Refs. [5,8]). An independent measurement of the $\mathrm{Fe}$ spin and orbital moments in the CFCT FePd would help to resolve this question. Another reason could be the fact that the measurements were conducted on the thin films of a few nanometers [4] where the surface/interface could play a significant role. Indeed, the MAE (about $0.26 \mathrm{meV} /$ cell) and total magnetic moment $\left(2.9 \mu_{\mathrm{B}} /\right.$ cell $)$ measured using conventional magnetometers in the films $[7,4]$ are somewhat smaller than that in the bulk materials [2,3] (Tables 1 and 2). Furthermore, the sum of the spin and orbital magnetic moments of $\mathrm{Fe}$ and $\mathrm{Pd}$ in the $\mathrm{Fe}_{0.5} \mathrm{Pd}_{0.5}$ alloy films determined using the XMCD technique (about $3.3 \mu_{\mathrm{B}} /$ cell) is greater than that by magnetometers [4]. Note that the computed total magnetic moments of FePd are in rather good agreement with total magnetization measurements on the bulk alloys [2,3] (Table 1).

The calculated MAE are listed in Table 2 which should be compared with Table 1 in Ref. [8]. Table 2 indicates that the use of GGA has no significant effect on the calculated MAE values. In contrast, Table 1 in Ref. [8] showed that the use of GGA increases the MAE of FePd in the EFCT structure by a factor of 10 on the one hand, and changes its sign for FePd in the CFCT structure on the other hand. Table 2 also demonstrates that like the computed $\mathrm{Fe}$ orbital moments, the computed MAE increases substantially upon the inclusion of OPC. This is consistent with the results of Daalderop et al. [5]. Furthermore, the MAE calculated with OPC are in good agreement with experiments [2,3] (Table 2). Note that the GGA result of Galanakis [8] differs qualitatively from experiments $[2,3,7,4]$.

Galanakis et al. [8] could argue that their results differ from that of the present work and also of Daalderop et al. [5] because the atomic sphere approximation (ASA) was used in the latter. However, the errors in the computed magnetic moments and MAE associated with ASA are expected to be small since the EFCT, FCC and CFCT structures are rather close-packed. Nevertheless, to check this possibility, we have also calculated the MAE of FePd in all the three structures using the highly accurate FLAPW method [12]. Again, we first performed self-consistent spin-polarized electronic structure calculations assuming the magnetization along [0 001 l]. The spin-orbit coupling was included in every iteration. The Brillouin zone integration was carried out with 180 inequivalent $k$-points. A large number of the augmented plane waves (APW) (about 110 per atom) (i.e.,

\section{Table 2}

Calculated magnetocrystalline anisotropy energies (MAE) of the ordered $\mathrm{Fe}_{0.5} \mathrm{Pd}_{0.5}$ alloy in the elongated (EFCT), ideal (FCC) and compressed (CFCT) $L 1_{0}$ structures. The MAE is

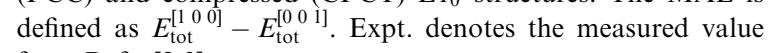
from Refs. [2,3]

\begin{tabular}{llll}
\hline MAE (mev) & EFCT & FCC & CFCT \\
\hline LSDA (LMTO) & 0.37 & 0.27 & 0.09 \\
LSDA-OPC (LMTO) & 0.69 & 0.64 & 0.52 \\
GGA (LMTO) & 0.37 & 0.27 & 0.04 \\
GGA-OPC (LMTO) & 0.66 & 0.59 & 0.41 \\
Expt. & & & $0.36(0.52)$ \\
LSDA (FLAPW) & 0.28 & 0.21 & 0.08 \\
GGA (FLAPW) & 0.29 & 0.21 & 0.06 \\
\hline
\end{tabular}

$R_{\mathrm{mt}} K_{\max }=9$ [12]) were included in the calculations. After the self-consistent calculations, the MAE was calculated with a larger number of $k$-points of 2000 plus a temperature broadening of $k_{\mathrm{B}} T=2 \mathrm{mRy}$. The resultant MAE are listed in Table 2. Clearly, the FLAPW results are in reasonable agreement with that obtained using the LMTO method. Note that the MAE computed with LSDA and GGA using the FLAPW method are nearly the same, again, in contradiction to that reported in Ref. [8].

Finally, lets discuss the possible origins of the surprising results presented in Ref. [8]. First of all, we note that the GGA calculations need both charge density gradients and charge densities. As a result, the GGA calculations would require a higher accuracy. This is because even small discontinuities in the charge density distribution, e.g., near the muffin-tin sphere surfaces can give rise to some pronounced errors in the calculated density gradients. For example, to get a smooth curve of energy vs. lattice constant for FePd using the FLAPW method [12], about 50 APWs per atom would be sufficient for the LSDA calculations while over $100 \mathrm{APW}$ would be needed for the GGA calculations. This would suggest that Galankis et al. should check the convergence in their GGA calculations since the same cut-offs used in the LSDA calculations might not be sufficient for the GGA calculations.

The authors thank the National Science Council of ROC for financial supports (NSC 88-2112-M002-0043, NSC 89-2112-M002-0025).

\section{References}

[1] F.C. Nix, W. Shockley, Rev. Mod. Phys. 10 (1938) 1.

[2] N. Miyata, et al., J. Phys. Soc. Jpn. 59 (1990) 1817.

[3] B. Zhang, W.A. Soffa, Scr. Metall. Mater. 30 (1994) 683.

[4] P. Kamp, et al., Phys. Rev. B 59 (1999) 1105. 
[5] G.H.O. Daalderop, P.J. Kelly, M.F.H. Shuurmans, Phys. Rev. B 44 (1991) 12054.

[6] I.V. Solovyev, P.H. Dederichs, I. Mertig, Phys. Rev. B 52 (1995) 13419.

[7] V. Gehanno, et al., Phys. Rev. B 55 (1997) 12552.

[8] I. Galanakis, et al., Phys. Rev. B 61 (2000) 599.

[9] M.S.S. Brooks, Physica B 130 (1985) 6.

[10] G.Y. Guo, Phys. Rev. B 55 (1997) 11619.

[11] H. Ebert, Phys. Rev. 38 (1988) 9391.
[12] P. Blaha, K. Schwarz, J. Luitz, WIEN97, Vienna University of Technology, 1997.

[13] S.H. Vosko, L. Wilk, M. Nusair, Can. J. Phys. 58 (1980) 1200.

[14] J.P. Perdew, S. Burke, M. Ernzerhof, Phys. Rev. Lett. 77 (1996) 3865.

[15] C.T. Chen, et al., Phys. Rev. Lett. 75 (1995) 152. 